\title{
AGING \\ Protein aggregation contributes to aging in C. elegans
}

Huang, C. et al. Elife 8, e43059 (2019)

Aging is associated with a progressive decline in many physiological functions, which decreases the quality of life of elderly individuals. The mechanisms underlying aging are still unclear, hampering the development of strategies to delay functional decline. By showing that protein aggregation accelerates the functional decline of different tissues in Caenorhabditis elegans, a new study provides viable targets for future strategies to delay tissue aging.

"Why do we age and more importantly, why do we experience functional decline with age? These are fundamental unresolved questions that we address in this present study," says lead investigator Della David. Her lab at the German Center for Neurodegenerative Diseases in Tübingen, Germany uses a combination of genetic, biochemical, proteomic, microfluidic, and high-resolution imaging approaches to examine age-dependent protein changes in C. elegans. C. elegans is a standard model organism for aging research because it offers advantages such as a short lifespan and readily available genetic tools. In 2010, David and colleagues showed that protein aggregation, a specific form of protein instability, is an inherent part of aging; however, important questions remained unanswered at that time. "We did not know if age-dependent protein aggregation influences the aging process. We were also lacking important structural information to assess how similar or not age-dependent protein aggregates are compared to protein aggregates in neurodegenerative diseases," explains David.

The toxic aggregation of proteins into amyloid fibrils is a hallmark of neurodegenerative diseases such as Alzheimer's disease. To determine if agedependent aggregates contain amyloid structures in the worms, the investigators used a fluorescence-lifetime based amyloid aggregation sensor first developed in 2011 by co-author Gabriele Kaminski Schierle from the University of Cambridge, UK. This non-invasive imaging technique can quantify the aggregation kinetics of amyloidogenic proteins in vivo. "The concept makes use of reporter fluorophores covalently linked to the protein of interest that are quenched upon aggregation. The method is now in world-wide use and brought significant new insights into

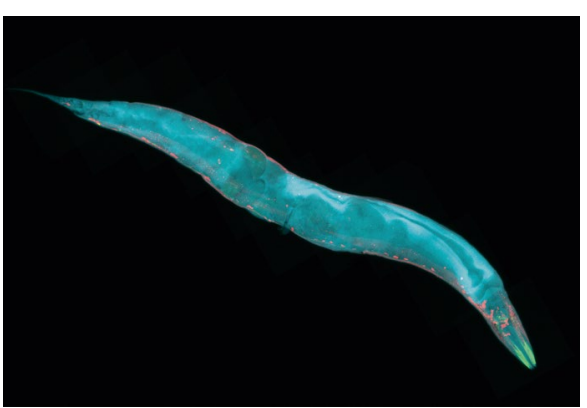

Caenorhabditis elegans. Fluorescence micrograph. Credit: Heiti Paves / Alamy Stock Photo

neurodegenerative diseases," explains Kaminski Schierle.

The investigators generated transgenic C. elegans expressing translational fusions of either Casein kinase I isoform alpha (KIN-19) or Ras-like GTP-binding protein rhoA (RHO-1) - two proteins with a high propensity to become insoluble with agingwith the fluorescent protein Venus. Timecorrelated single photon counting lifetime imaging revealed that KIN-19::Venus and RHO-1::Venus had a significantly decreased fluorescence lifetime compared with Venusonly control worm, confirming the intrinsic capacity of these two proteins to aggregate into amyloid-like structures, similar to disease-associated proteins.

Next, the investigators evaluated whether protein aggregation contributes to functional decline by measuring the effects of fluorescent-labelled KIN-19 and RHO-1 overexpression on pharyngeal pumping and swimming, two physiological processes that decline with age. First, analysis of fluorescence distribution confirmed that KIN-19 and RHO-1 aggregation increased with age in all transgenic animals. Pharyngeal electrical activity and swimming were then recorded to compare pharyngeal pumping and swimming in the different animals. Analysis revealed that overexpression and aggregation of KIN-19 in the body-wall muscles of aged worms significantly reduced swimming frequency compared with wild-type worms or control worms overexpressing only a fluorescent protein, and high levels of KIN-19 aggregation in the pharynx were associated with an earlier decline in pumping frequency. To further show that protein aggregation causes functional decline, independently of other aging factors, the effects of RHO-1 overexpression were investigated in young animals. Overexpression led to protein aggregation, which reduced the frequency of pharyngeal pumping in young worms to rates normally observed in aged animals. Altogether these result showed that increased protein aggregation accelerates functional decline in C. elegans. "Unlike previous studies showing only correlative evidence, our results provide a causal relationship. Aggregation of proteins during normal aging is not an irrelevant side-effect of aging, rather it is likely to be one of the driving causes of functional decline," says David.

David adds that the team is now focusing on finding endogenous mechanisms in C. elegans that could protect against agedependent protein aggregation. For instance, alterations in protein homeostasis caused by a reduction in molecular chaperones linked to protein synthesis or impaired proteasomemediated removal of unfolded proteins directly after synthesis might contribute to age-dependent protein aggregation; identifying mechanisms and pathways that counteract the effects of these alterations might provide new target for strategies to delay functional decline associated with aging.

The team is also looking into ways to improve the sensitivity and throughput of their amyloid aggregation sensor. "In order to better screen for the level of protein aggregation and thus for drugs that can alleviated the latter in live organisms, we are also currently investigating how to integrate the fluorescence lifetime-based platform into a more high-throughput imaging platform," says Kaminski Schierle, adding that they would be open to collaborations with companies.

Future areas of investigation also include exploring how age-dependent protein aggregation is linked to unhealthy aging and neurodegeneration. "The structural similarity between age-dependent protein aggregates and disease-associated protein aggregates raises questions about the nature of their interaction and how they influence each other's aggregation," conclude the investigators.

Alexandra Le Bras

Published online: 10 June 2019

https://doi.org/10.1038/s41684-019-0335-5 